Originalveröffentlichung in: Leutner, Petra ; Niebuhr, Hans-Peter (Hrsgg.): Bild und Eigensinn : über Modalitäten der Anverwandlung von Bildern, Bielefeld 2006,

S. 149-159 (Kultur- und Medientheorie)

\title{
»WIE DIE ANSTALT SIE HABEN MÖCHTE ...« - SELBSTBILdER IN DER SAMMLUNG PRINZHORN
}

\author{
THOMAS RÖSKE
}

Die Sammlung Prinzhorn an der Psychiatrischen Universitätsklinik Heidelberg ist seit 2001 öffentlich zugänglich: als ein Museum für künstlerische Werke vorwiegend von Anstaltsinsassen und von, wie wir heute sagen, Psychiatrieerfahrenen. ${ }^{1}$ Sein wichtigster Besitz sind mehr als fünftausend Malereien, Zeichnungen, Skulpturen und Textilarbeiten, die nach dem Ersten Weltkrieg von einer Vielzahl psychiatrischer Anstalten, Sanatorien und Kliniken vor allem deutschsprachiger Länder nach Heidelberg geschickt wurden - auf einen Aufruf des Kunsthistorikers und Mediziners Hans Prinzhorn (1886-1933) hin. Er war 1919 als Assistenzarzt berufen worden, um eine bereits bestehende kleine Sammlung zu erweitern und in einer wissenschaftlichen Studie auszuwerten. Sein Buch Bildnerei der Geisteskranken. Ein Beitrag zur Psychologie und Psychopathologie der Gestaltung erschien $1922,{ }^{2}$ ein Jahr, nachdem Prinzhorn die Klinik verlassen hatte. Der für die Zeit ungewöhnlich prachtvolle Band enthielt nicht nur die umfangreichste Erörterung von Fragestellungen um »Irrenkunst«; er machte das Gebiet, das heute der so genannten Outsider Art zugerechnet wird, mit seinen 170 Abbildungen auch erstmals für ein großes Publikum sichtbar. Erfolg hatte es wesentlich bei Künstlern und Kunstinteressierten. ${ }^{3}$ Als ,Klassiker wurde es bis heute mehrfach wiederaufgelegt.

In der Zeit, aus der die historischen Werke der Sammlung Prinzhorn stammen, den Jahrzehnten zwischen 1880 und 1930, hatten psychische

* Ich danke für eine Diskussion meines Vortrags und für weiterführende Hinweise den Teilnehmern des Offenbacher Symposions und Dr. Bettina Brand-Claussen.

1 Siehe Rückblick: Sammlung Prinzhorn - Rückblick 2001-2005, hg. von Thomas Röske, Bettina Brand-Claussen und Monika Jagfeld, Heidelberg: Sammlung Prinzhorn 2005.

2 Hans Prinzhorn: Bildnerei der Geisteskranken. Ein Beitrag zur Psychologie und Psychopathologie der Gestaltung, Berlin: Springer 1922.

3 Thomas Röske: Der Arzt als Künstler. Ästhetik und Psychotherapie bei Hans Prinzhorn (1886-1933), Bielefeld: Aisthesis 1995. 
Krisen andere Folgen als heute. Die Toleranz gegenüber einem Abweichen von snormalem< Verhalten war geringer, die Stigmatisierung von , Verrücktheit stärker. Instruktiv ist das Gegenüber zweier Fotografien einer Frau, die Eintritt und Austritt dokumentieren sollten, nach dem Prinzip des > Vorher - Nachher . Die Fotos sind in einem Album bewahrt, das um 1910 in der hessischen Anstalt Weilmünster zur Ausbildung des Personals angelegt wurde (Abb. 1). ${ }^{4}$

Abbildung 1: ,Vorher-Nachher. Anonyme Fotografien aus der Anstalt Weilmünster 1905-1914

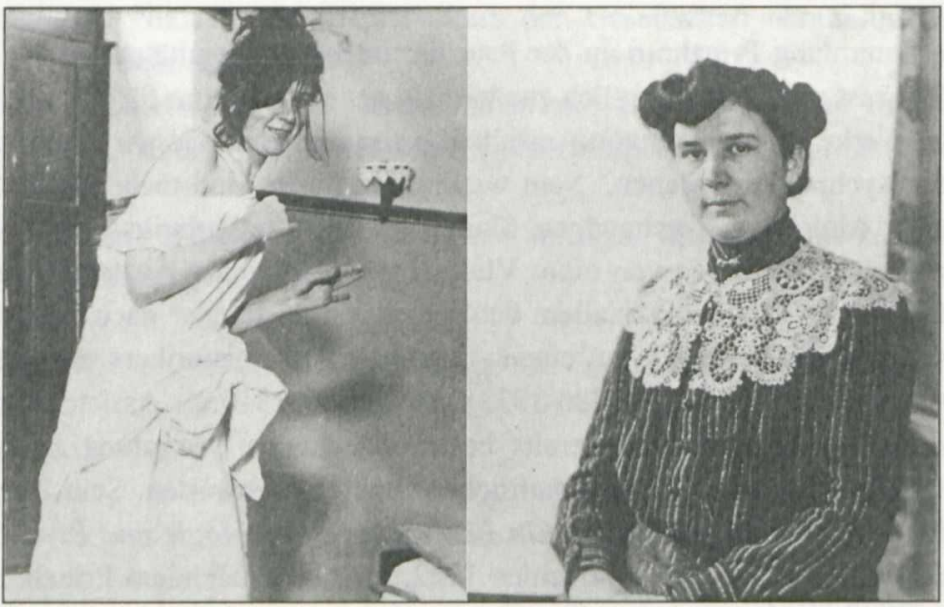

Die Bürgersfrau im Zustand der Manie erscheint uns heute sgesünder,, glücklicher, freier als in ihrer gehemmten und angepassten Erscheinung beim Verlassen der Anstalt. Tatsächlich waren die Psychiater damals hilfloser als heute. Während dieser Tage die meisten, die eine psychische Krise durchleben, nur kurz stationär behandelt, das heißt mit Hilfe verschiedener Therapien und Medikamente resozialisiert werden, blieben damals die meisten Menschen mit der Diagnose Dementia praecox (vorzeitige Verblödung) - ab 1911 allmählich Schizophrenie - auf Dauer interniert. Sie wurden oft dreißig bis vierzig Jahre lang bis zu ihrem Tod weggeschlossen in Institutionen, wo man außer Bettbehandlung, Beruhigungsmitteln, Zwangsmaßnahmen (Dauerbad, Fixierungen, Tobzelle) und Arbeitstherapie keine Behandlungsmöglichkeiten kannte. Die Folge

$4 \mathrm{Zu}$ diesen Alben vgl.: Ins Gesicht sehen. Band 1: Anonyme Fotografien aus der Anstalt Weilmünster 1905-1914, hg. von Bettina Brand-Claussen und Thomas Röske, Ausstellungskatalog Sammlung Prinzhorn Heidelberg, Heidelberg: Sammlung Prinzhorn 2002. 
sozialer und intellektueller Unterstimulierung war fast immer eine Art Autismus, den man fälschlich als zwangsläufigen »schizophrenen Endzustand« deutete. Auch die historischen Werke der Sammlung Prinzhorn stammen vielfach von Langzeitpatienten, die sich kaum noch sprachlich äußerten. Anders als Prinzhorn sehen die wissenschaftlichen Mitarbeiter des Museums heute weniger Eruptionen des Unbewussten darin, sondern versuchen, durch biographische Recherche und kultur- und sozialhistorisches Kontextualisieren den Mitteilungscharakter der Werke offen zu legen.

Besonders sprechend können in dieser Hinsicht Selbstdarstellungen sein, wobei sich die von männlichen und weiblichen Anstaltsinsassen in charakteristischer Weise unterscheiden. Einige hat auch Prinzhorn unter die Illustrationen seines Buches aufgenommen. Allerdings schien ihm ein »bildnerische(s) Selbstbekenntnis« im »vollen Sinne des Wortes« allein der »Könner« Franz Karl Bühler (1864-1940) (von ihm »Franz Pohl« genannt) geschaffen zu haben, »der seine Wortsprache längst nur noch zu verschrobenen Spielereien benutzt. $\ll^{5}$ Der Farbstiftzeichnung von 1918 widmete er eine ganze Seite (Abb. 2).

Hier hat sich der Kunstschmied, der sich in der Zeit seines Anstaltsaufenthaltes ab 1898 zum freien Künstler entwickelte, in einem Büstenausschnitt dargestellt, »den Kopf leicht vorgeneigt, mit runden, lebhaft herausschauenden Augen«. Prinzhorn vergleicht dieses Porträt mit Vincent »van Goghs späte(m) Selbstbildnis«: »nur dort treffen wir einen Menschen, der in so brennender Spannung hinausschaut und dabei so trostlos zerstört in seinem Weltgefühl zu sein scheint. « ${ }^{6}$

Was die Qualität des Blattes herausstellen soll, macht vor allem den Maßstab des Urteilenden und die Grundlage seiner Sicht auf »Bildnerei der Geisteskranken« deutlich: Prinzhorns Geschmack ist vom Spätimpressionismus und Expressionismus geprägt. ${ }^{7}$ Und von der Authentizität (»Echtheit«) der »Irrenkunst« ist er überzeugt, weil für ihn die ähnlichen Werke van Goghs die unmittelbare Nachbarschaft von Genie und Wahnsinn belegen. Prinzhorn folgt also bei seinem Wertschätzen des Unkonventionellen Konventionen seiner Zeit. Das zeigt sich bei dieser Zeichnung auch in seinem Schweigen über andere ihrer gestalterischen Verfahren. Auffällig ist, dass Bühlers Büste Aufschriften verschiedener GröBe und Type umgeben, Mitteilungsfragmente, die eine für uns inkohären-

5 Prinzhorn, Bildnerei, S. 286.

6 Ebd.

7 Bettina Brand-Claussen: »Prinzhorns >Bildnerei der Geisteskranken spätexpressionistisches Manifest $\lll$, in: Vision und Revision einer Entdeckung, hg. von Inge Jádi und Bettina Brand-Claussen, Ausstellungskatalog Sammlung Prinzhorn Heidelberg, Heidelberg: Sammlung Prinzhorn 2001, S. 11-31. 
te Botschaft bilden - Repräsentanten zerfallenden Sinns, aus denen der Künstler mit leicht gesenktem Kopf hervorschaut: Vor allem die ausgemalte, »offizielle« Fraktur betont die Fläche, die sein Kopf in unsere Richtung durchbricht, als wolle er mit seiner leicht demütigen Haltung und seinem traurig fragenden Blick dem Betrachter näher kommen, als (ihm) dies mit Sprache (noch) möglich ist.

Abbildung 2: Franz Karl Bühler, o.T. (Selbstporträt), 1918, Farbstifte und Kreide, z.T. laviert, auf Zeichenpapier, 27,7 x 18,9 cm, Inv.Nr. 2987.

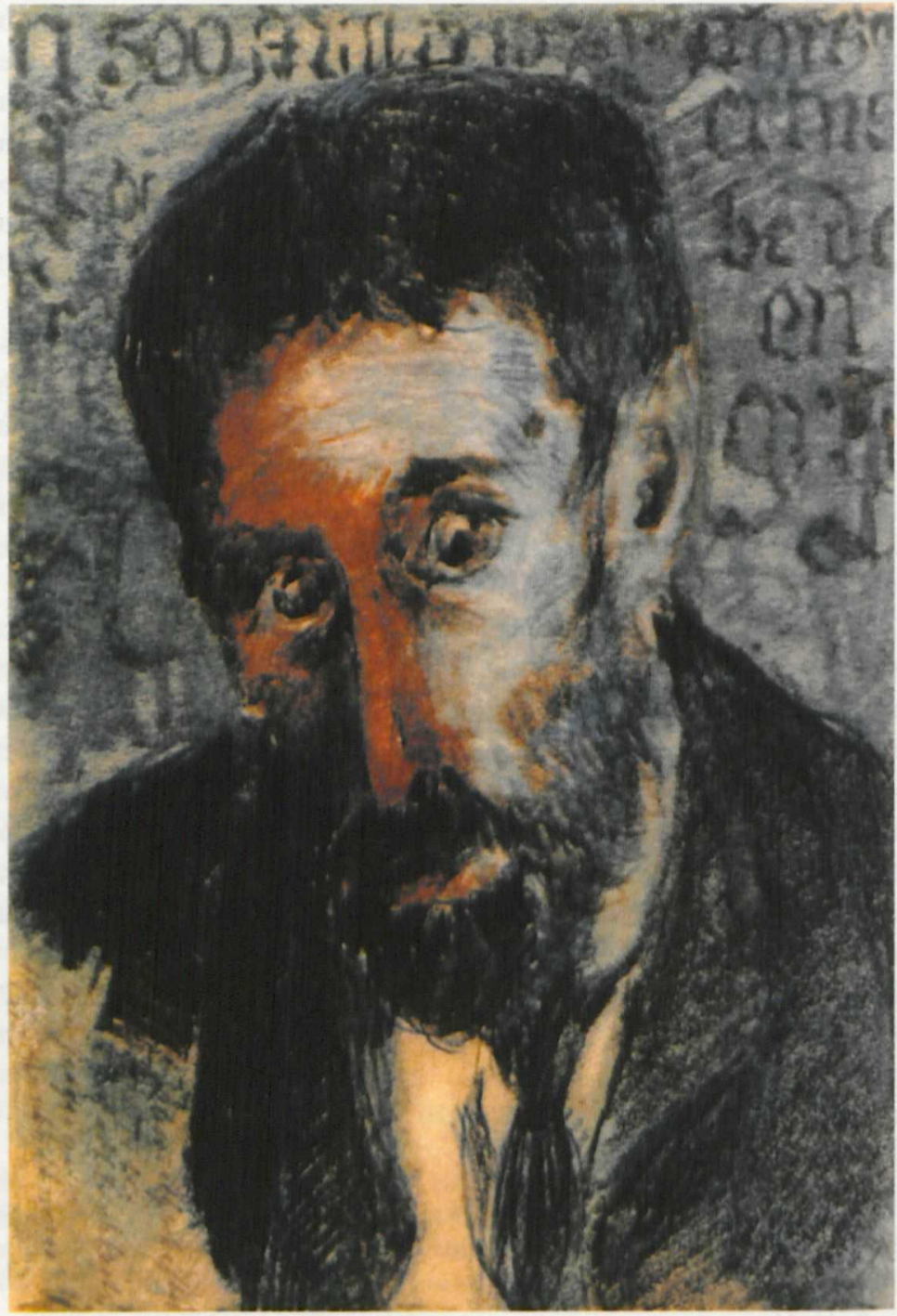


Weitere Selbstdarstellungen Bühlers belegen ebenfalls, dass ihm gerade an einem Problematisieren von Konventionen gelegen war. So zeigt er sich zum Beispiel einmal (Abb. 3) mit einem mehrdeutigen länglichen Gegenstand in Händen, den er zum Mund geführt hat.

Abbildung 3: Franz Karl Bühler, o.T. (Selbstbildnis), um 1909, Kreide auf Papier, 41,5 $30,9 \mathrm{~cm}$, Sammlung Prinzhorn Heidelberg, Inv.Nr. 2831.

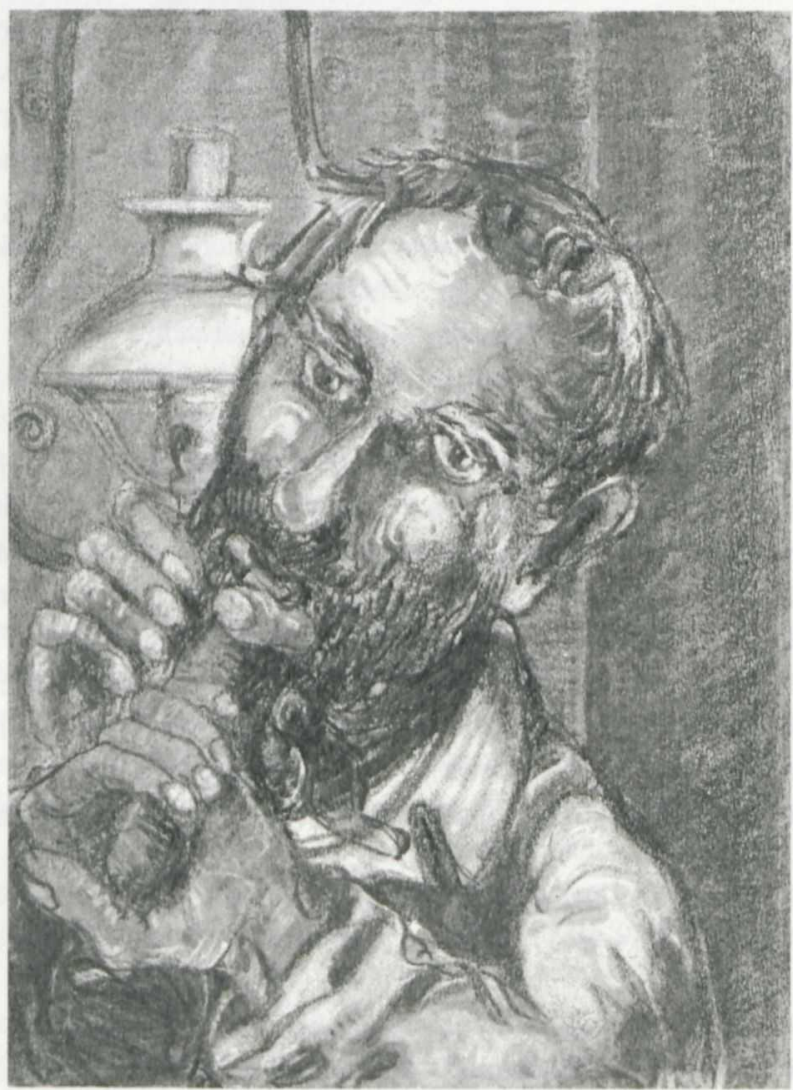

Zunächst scheint es, dass er Flöte spielt, zumal seine Backen aufgeblasen sind und sein Blick auseinander oder ins Leere geht, wie bei jemandem, der sich auf Klang konzentriert. Es gibt noch andere Zeichnungen Bühlers mit Musikern, auf einer hält er auch zwei Querflötenspieler fest. ${ }^{8}$ Sie

8 Franz Karl Bühler, o.T. (Flötenspieler), 1920, Bleistift, Sammlung Prinzhorn, Inv.Nr. 3025, fol. 13, abgebildet in: Franz Karl Bühler (Offenburg 1864-Grafeneck 1940). Bilder aus der Prinzhorn-Sammlung, Ausstellungs- 
belegen zugleich, dass er wusste, wie man Musikinstrumente gebraucht. Daher greift er sicherlich nicht aus Unkenntnis die Längsflöte verkehrt: Seine Linke hält er umständlich mit dem Rücken nach unten. Ist das, was er hält, aber wirklich eine Flöte? Ist es nicht eher eine Karotte oder - das vordere Ende sieht aus wie eine Eichel - ein männliches Geschlechtsteil? Man könnte an den umgangssprachlichen Ausdruck für Fellatio denken: jemandem einen blasen; auch das Wenden nach links und der geringere Platz dort sprechen für eine intime, der Öffentlichkeit entzogene Handlung. Blickt man auf die Leidensgeschichte Bühlers, erhält dieser Verweis auf eine homosexuelle Praktik noch eine weitere Dimension: Die Verfolgungsideen, die ihn schließlich in die Anstalt brachten, entwickelte er nach seiner Entlassung aus der Kunstgewerbeschule in Straßburg 1896, die er als tiefe Kränkung und traumatischen Einschnitt seiner Biographie erlebte. ${ }^{9}$ Bühler scheint sich auf dieser Kreidezeichnung als jemanden darzustellen, der den Phallus, die männliche Rolle innerhalb der Gesellschaft nur ungelenk zu gebrauchen weiß, der marginalisiert wurde, weil er die Spielregeln der Gesellschaft nicht beherrschte.

Männer und Frauen wurden um 1900 auch in psychiatrischen Anstalten unterschiedlich behandelt. ${ }^{10}$ Bei Männern war Gesundungsziel das verantwortliche und arbeitsfähige gesellschaftliche Subjekt - für das Leiden unter Defiziten in dieser Richtung sind gerade Bühlers Selbstporträts gute Beispiele. Demgegenüber sollte die verrückte Frau affektiv korrigiert und in die Rolle des angepassten Objekts zurückgeführt werden. Tatsächlich lassen die Krankenakten erkennen, dass junge Frauen damals nicht selten deshalb in psychiatrische Behandlung gegeben wurden, weil sie gegen gesellschaftliche Erwartungen verstoßen hatten. Der Anpassungsdruck auf Frauen war größer. Das zeigt sich auch in Selbstdarstellungen von Psychiatriepatientinnen, wie zwei Beispiele belegen sollen.

katalog Museum im Ritterhaus, Offenburg: Museum im Ritterhaus 1993, S. 123.

9 Zu Bühler vgl. zuletzt: Monika Jagfeld: „Geistertänzer. Franz Karl Bühler Ein 〉Geisteskranker als Expressionist? «, in: Expressionismus und Wahnsinn, Ausstellungskatalog Stiftung Schleswig-Holsteinische Landesmuseen, Schloß Gottorf, München: Prestel 2003, S. 88-94.

10 Siehe hierzu Maike Rotzoll u.a.: »Frauenbild und Frauenschicksal - Weiblichkeit im Spiegel psychiatrischer Krankengeschichten zwischen 1900 und $1940 \ll$, in: Irre ist weiblich - Künstlerische Interventionen von Frauen in der Psychiatrie, hg. von Bettina Brand-Claussen und Viola Michely, Ausstellungskatalog Sammlung Prinzhorn Heidelberg, u.a., Heidelberg: Wunderhorn 2004, S. 45-52. 
Ihre Wahrnehmung des Verhältnisses von Arzt und Patientin setzt Minna Köchler (1886 - nach 1920) 1906 ins Bild (Abb. 4). ${ }^{11}$

Abbildung 4: Minna Köchler, Sie Selbst/Herr Dr. Wilmanns, 1906, Bleistift auf Aktenpapier, 33,0 × 21,0 cm, Sammlung Prinzhorn Heidelberg, Inv.Nr. $4067 \mathrm{fol} . \mathrm{Iv}$.

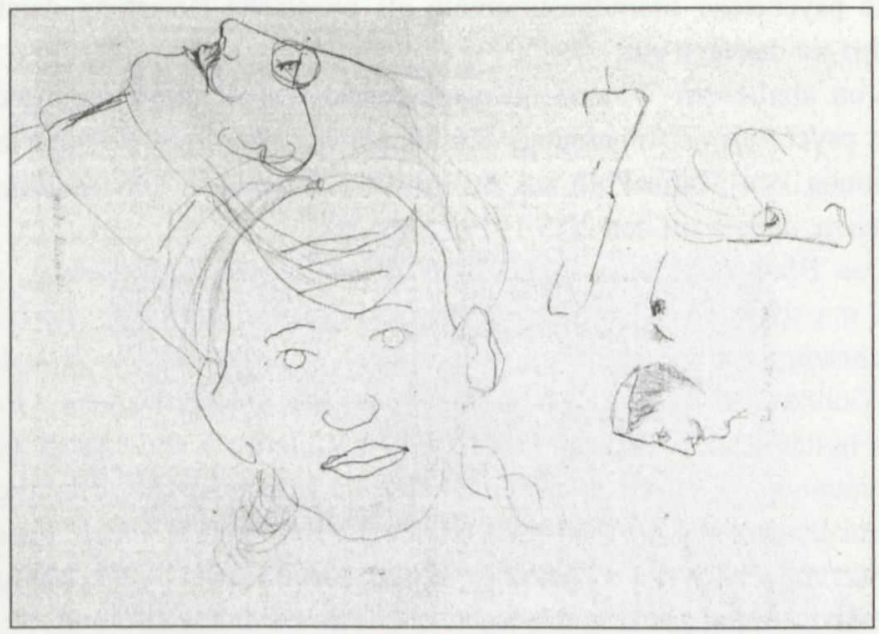

Auf das Blatt im Querformat hat sie ihr eigenes Gesicht gezeichnet - von fremder Hand steht »Sie Selbst« darunter. Wenige sichere Linien charakterisieren die sanften Augen, die weiche Nasenspitze und den geschlossenen sinnlichen Mund. Die Züge wirken entspannt, nur auf der Stirn, unter dem Ansatz des zurückgekämmten (hinten zu Zöpfen geflochtenen) Haars, stehen drei Falten. Dass man sie nicht gleich sieht, liegt an einer Irritation der Zeichnung. Sie wird hier überlagert von dem kleineren Kopf eines Mannes im Profil, der im Winkel von 90 Grad zum Selbstbildnis steht. Köchler hat das Blatt beim Zeichnen auf die Seite gedreht. Bei dem Brillenträger mit dem gezwirbelten Schnurrbart und dem hohen steifen Kragen handelt es sich, so die Beischrift, um »Herr(n) Dr. Wilmanns « - laut Krankenakte damals ihr behandelnder Arzt an der Heidelberger Psychiatrischen Universitätsklinik (nach dem Weltkrieg wird er deren Direktor und hat die Idee zu dem heute nach Prinzhorn benannten Sammlungsprojekt). Mit dem Gegensatz von en face und Profil und dem unterschiedlichen Ausrichten des Blattes betont Köchler gestalterisch die Distanz zwischen ihr und dem Arzt. Die Überlagerung der beiden Dar-

$11 \mathrm{Zu}$ Minna Köchler vgl.: Irre ist weiblich, Ausst. Kat. Heidelberg 2004, S. $257 \mathrm{f}$. 
stellungen scheint zunächst zufällig, zumal Köchler sich auch anderswo auf dem Blatt an diesem Männerprofil versucht hat. ${ }^{12}$ Doch macht eine Korrektur stutzig, mit welcher die Kontur des männlichen Hinterkopfes zweimal vergrößert wurde. Denn gerade diese Dreierlinie reicht in das Selbstbildnis Köchlers hinein und korrespondiert hier zu den drei Falten über den Augen. Die Zeichnerin reagiert mit Stirnrunzeln auf das schrittweise Vordringen des Arztkopfes in den ihren. Indem sie eine erspürte psychische Fremdbestimmung als physische Belastung darstellt, begehrt sie dagegen auf.

Von ähnlichem Widerstand gegen einen von der Institution ausgeübten psychischen (Anpassungs-)Druck spricht eine aquarellierte Pinselzeichnung von Maria Puth aus der privaten Kuranstalt Obersendling in München, datiert auf den 20.3.1919 (Abb. 5). ${ }^{13}$

Das Blatt zeigt eine Frauenfigur in einem weich fließenden rosa Kleid mit schwarzen Punkten, die Hut und Stockschirm trägt. Die feinen Gesichtszüge wirken ernst, die Augen sind geschlossen. Die Gestalt ist von Graulavierungen umgeben und steht auf grauschwarzem Grund. Links hinten deuten mehrere Pinselzüge in Violettrosa und Orange einen Sonnenuntergang an. Lockere Bleistiftstriche fassen die Darstellung ganz ein, nur über dem Kopf ist dieser Rahmen unterbrochen. Oben links hat ein Arzt mit Feder seine Diagnose notiert: „M.P., geb. 1.7.94. Manischdepressiv. Heitere Erregung mit kurzen Depressionen.« Puth selbst hat das Bild unten auf dem Blatt kommentiert: »Ria Puth, wie die Anstalt sie haben möchte, sie aber scala Dei (sc. )leider Gottes $`$ ) nie wird.«

Dies ist ein sprechendes Gegenbeispiel zu Prinzhorns These von der unbewussten bildnerischen Gestaltung von Psychiatriepatienten, ein Blatt, das er wohl schon deshalb, aber auch wegen seiner schlichten Gegenständlichkeit nicht abgebildet hat. Puth dreht unter den Augen ihrer Ärzte eine höchst originelle bildnerisch-argumentative Pirouette. Sie zeigt, dass sie weiß, welche innere wie äußere Haltung der Anstalt für sie vorschwebt - wobei sie der Darstellung einer modisch gekleideten und verhalten auftretenden Frau deutlich traurige Züge verleiht (eine depressive Grundstimmung macht nicht zuletzt gefügig oder ist die Folge derartiger Gefügigkeit). Auf anderer Ebene, der des begleitenden Textes, distanziert sie sich von dem locker gestalteten Selbstbild und verweist es ins Irreale. Das verballhornte Latein der Wendung »scala Dei $(=»$ Leiter Gottes«) gibt Ironie zu erkennen: Gegen die Anpassung an das entworfene Bild steht nicht eine äußere, ihrem Willen entzogene Macht, son-

12 Obgleich am rechten Bildrand ein anderer Name aufgezeichnet wurde, scheint es sich stets um den Kopf desselben Mannes zu handeln.

13 Zu Maria Puth vgl.: Doris Noell-Rumpeltes: »Maria Puth«, in: Irre ist weiblich, Ausst. Kat. Heidelberg 2004, S. 196. 
dern allein sie selbst. Das Blatt von Maria Puth macht deutlich, dass Anstaltsinsassen durchaus in der Lage waren, die komplexen Machtverhältnisse innerhalb dieser Institutionen zu reflektieren.

Abbildung 5: Maria Puth, Wie die Anstalt sie haben möchte ..., 1919, Deckfarbe über Bleistift auf Zeichenpapier, $24,2 \times 14,9 \mathrm{~cm}$, Sammlung Prinzhorn Heidelberg, Inv.Nr. 2544

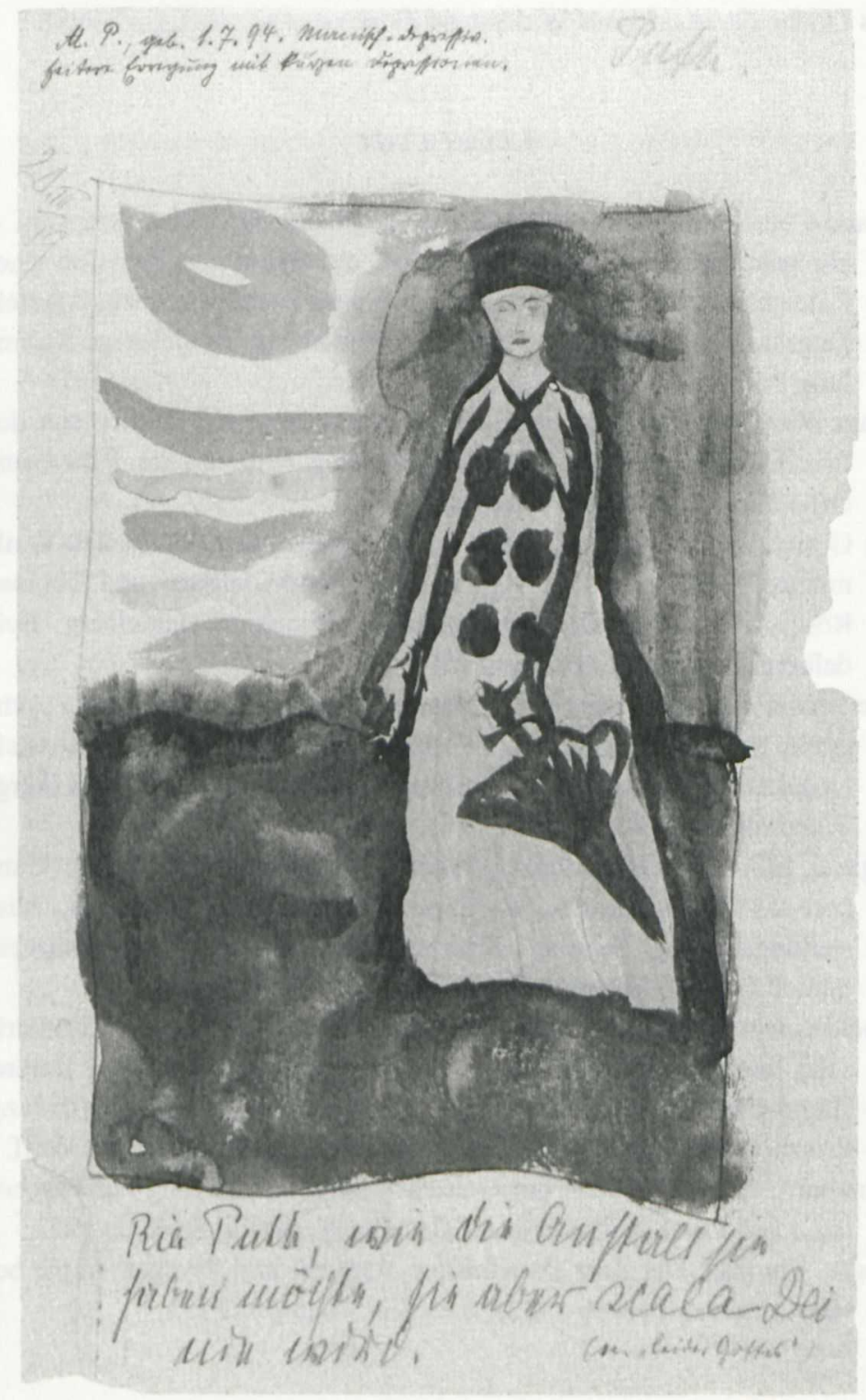


Wie an diesen Beispielen deutlich wurde, können bildnerische Äußerungen von Insassen psychiatrischer Anstalten auf »Eigensinn « besonderen Anspruch erheben - und das nicht so sehr deshalb, weil ihre Wahrnehmung, ihr Denken und Empfinden vollkommen anders als das der Menschen außerhalb der Anstaltsmauern wäre, wie Prinzhorn im Konsens mit der damaligen Psychiatrie meinte. Vielmehr zeigt sich in vielen dieser Werke gerade auch die Auseinandersetzung mit einer erlebten Differenz zu den Erwartungen der umgebenden Gesellschaft, motiviert entweder aus Leiden daran oder aus Widerstand dazu.

\section{Literatur}

Brand-Claussen, Bettina: »Prinzhorns `Bildnerei der Geisteskranken « ein spätexpressionistisches Manifest«, in: Vision und Revision einer Entdeckung, hg. von Inge Jádi und Bettina Brand-Claussen, Ausstellungskatalog Sammlung Prinzhorn Heidelberg, Heidelberg: Sammlung Prinzhorn 2001, S. 11-31.

Franz Karl Bühler (Offenburg 1864-Grafeneck 1940). Bilder aus der Prinzhorn-Sammlung, Ausstellungskatalog Museum im Ritterhaus, Offenburg: Museum im Ritterhaus 1993.

Ins Gesicht sehen. Band 1: Anonyme Fotografien aus der Anstalt Weilmünster 1905-1914, hg. von Bettina Brand-Claussen und Thomas Röske, Ausstellungskatalog Sammlung Prinzhorn Heidelberg, Heidelberg: Sammlung Prinzhorn 2002.

Irre ist weiblich - Künstlerische Interventionen von Frauen in der Psychiatrie, hg. von Bettina Brand-Claussen und Viola Michely, Ausstellungskatalog Sammlung Prinzhorn Heidelberg, u.a., Heidelberg: Wunderhorn 2004.

Jagfeld, Monika: »Geistertänzer. Franz Karl Bühler - Ein `Geisteskranker als Expressionist?«, in: Expressionismus und Wahnsinn, Ausstellungskatalog Stiftung Schleswig-Holsteinische Landesmuseen, Schloß Gottorf, München: Prestel 2003, S. 88-94.

Noell-Rumpeltes, Doris: »Maria Puth«, in: Irre ist weiblich - Künstlerische Interventionen von Frauen in der Psychiatrie, hg. von Bettina Brand-Claussen und Viola Michely, Ausstellungskatalog Sammlung Prinzhorn Heidelberg, u.a., Heidelberg: Wunderhorn 2004, S. 196ff.

Prinzhorn, Hans: Bildnerei der Geisteskranken. Ein Beitrag zur Psychologie und Psychopathologie der Gestaltung, Berlin: Springer 1922.

Röske, Thomas: Der Arzt als Künstler. Ästhetik und Psychotherapie bei Hans Prinzhorn (1886-1933), Bielefeld: Aisthesis 1995. 
Rotzoll, Maike u.a.: »Frauenbild und Frauenschicksal - Weiblichkeit im Spiegel psychiatrischer Krankengeschichten zwischen 1900 und 1940«, in: Irre ist weiblich - Künstlerische Interventionen von Frauen in der Psychiatrie, hg. von Bettina Brand-Claussen und Viola Michely, Ausstellungskatalog Sammlung Prinzhorn Heidelberg, u.a., Heidelberg: Wunderhorn 2004, S. 45-52.

Rückblick: Sammlung Prinzhorn - Rückblick 2001-2005, hg. von Thomas Röske, Bettina Brand-Claussen und Monika Jagfeld, Heidelberg: Sammlung Prinzhorn 2005. 